\title{
Two theorems on harmonic manifolds
}

\author{
Y. Nikolayevsky*
}

\begin{abstract}
A Riemannian manifold is called harmonic, if for any point $x$ it admits a nonconstant harmonic function depending only on the distance to $x$. A.Lichnerowicz conjectured that any harmonic manifold is two-point homogeneous. This conjecture is proved in dimension $n \leq 4$ and also for some classes of manifolds, but disproved in general, with the first counterexample of dimension 7. We prove the Lichnerowicz Conjecture in dimension 5: a five-dimensional harmonic manifold has constant sectional curvature. We also obtain a functional equation for the volume density function $\theta(r)$ of a harmonic manifold and show that $\theta(r)$ is an exponential polynomial, a finite linear combination of the terms of the form $\operatorname{Re}\left(c e^{\lambda r} r^{m}\right)$, with $c, \lambda$ complex constants.
\end{abstract}

Mathematics Subject Classification (2000). 53C25.

Keywords. Harmonic manifold, volume density.

\section{Introduction}

A Riemannian manifold $M$ is called harmonic if for any point $x \in M$ there exists a nonconstant harmonic function defined on a punctured neighbourhood of $x$ and depending only on the distance to $x$. Equivalently, for any point $x \in M$ the volume density function $\theta_{x}=\sqrt{\operatorname{det} g_{i j}}$ (in normal coordinates centered at $x$ ) is radial, that is, depends only on the distance to $x$; the mean (the scalar) curvature of a small geodesic sphere depends only on its radius (see [2, Ch. 6; 1], [Ch. 2.6; 12] for other equivalent definitions).

Two-point homogeneous spaces are harmonic. In 1944, Lichnerowicz conjectured that the converse is true: any harmonic space is two-point homogeneous. This conjecture is proved in dimension $\leq 4$ [15], for compact simply connected manifolds and for Ricci-flat manifolds [13], [14], for negatively curved compact manifolds [3], and also for some other classes of manifolds. However, in 1992, Damek and Ricci discovered a class of harmonic non-compact spaces, which are, in general, not symmetric, hence disproving the Lichnerowicz Conjecture [4]. For an account of results on harmonic spaces and Damek-Ricci spaces we refer to [1], [14], [17].

\footnotetext{
*Work supported by the ARC grant S6005288.
} 
The lowest dimension of a non-symmetric Damek-Ricci space is 7, and one might wonder if the Lichnerowicz Conjecture is true in dimension $n=5,6$. A partial answer is given by the following theorem:

Theorem 1. A five-dimensional harmonic space has constant curvature.

A similar result, under an assumption of pinched curvature, was obtained in [16].

On a harmonic manifold, the infinite sequence of algebraic conditions, the Ledger formulae, on the curvature tensor and its covariant derivatives must hold [2, Ch. 6 $\S \mathrm{C}]$. The first two of them are

$$
\operatorname{Ric}(X, X)=\operatorname{Tr} R_{X}=C\|X\|^{2}, \quad \operatorname{Tr}\left(R_{X}\right)^{2}=H\|X\|^{4},
$$

where $R_{X}$ is the Jacobi operator defined by $R_{X} Y=R(X, Y) X$, and the functions $C$ and $H$ are constant on the manifold. A Riemannian manifold satisfying (1) is called 2-stein (see, e.g. [6]).

Theorem 1 follows from Proposition 1 below and the fact that harmonic symmetric spaces are two-point homogeneous [5], [9].

Proposition 1. A five-dimensional 2-stein Riemannian manifold is either of constant curvature or is locally homothetic to the symmetric space $\mathrm{SU}(3) / \mathrm{SO}(3)$ or to its noncompact dual SL(3)/ SO(3).

One of the main ingredients of the proof of the Lichnerowich conjecture in the compact simply-connected case [13] is the fact that the volume density function is a trigonometric polynomial of a special structure. Moreover, the volume densities of Damek-Ricci spaces (including non-compact ROSS's) are polynomials of $\cosh r$ and $\sinh r$. We prove the following theorem.

Theorem 2. The volume density function of a harmonic manifold is an exponential polynomial: a finite linear combination of the terms of the form $\operatorname{Re}\left(c_{i} e^{\lambda_{i} r} r^{m_{i}}\right)$, with $c_{i}, \lambda_{i}$ complex constants.

This gives a partial answer to the question asked in [14]: what functions may occur as volume densities of harmonic spaces? Note that, in general, nonisometric harmonic spaces may have the same volume density function. However, in many cases, the volume density function determines a harmonic space uniquely: a harmonic space having the same volume density as that of one of the spaces $H^{n}, \mathbb{C} H^{n}, \mathbb{H} H^{n}$, is isometric to it, provided it is Kähler or quaternionic Kähler in the last two cases, respectively ([10]; the same is true under weaker assumptions: a manifold is Einstein and has the same volume growth of geodesic balls as that of the corresponding model space $[7, \mathrm{Sec} .8,9])$. A harmonic space with a polynomial volume growth is flat [12]. 
Note that Theorem 2 combined with the approach of [11] gives an alternative proof of the Lichnerowich conjecture in the compact case.

Theorem 2 will follow from the functional equation (2) below.

Let $\gamma(t)$ be a parameterized geodesic on a Riemannian manifold $M$, and denote $x=\gamma(0), T=\dot{\gamma}(0), L=T^{\perp} \subset T_{x} M$. For vectors $X, Y \in L$, let $J_{X, Y}(t)$ be the Jacobi field along $\gamma$ such that $J_{X, Y}(0)=X$ and $\nabla_{T} J_{X, Y}=Y$. Define the operator $\boldsymbol{Q}(t): L \rightarrow L$ by the formula $J_{0, \boldsymbol{Q}(t) X}(t)=J_{X, 0}(t)$ for $t \in\left(0, t_{1}\right)$, where $t_{1}$ is the distance to the first conjugate point on $\gamma$. The operator $\boldsymbol{Q}(t)$ is symmetric, with the asymptotic expansion $t^{-1} \mathrm{id}_{L}-\frac{1}{3} t R_{T}+o(t)$ at $t=0$, where $R_{T}: L \rightarrow L$ is the Jacobi operator. Extending the density function $\theta(t)$ to negative values of $t$ (by setting $\theta(-t)=(-1)^{n-1} \theta(t)$ for $\left.t>0\right)$ we have the following proposition.

Proposition 2. On a harmonic manifold, for $t, s \in\left(0, t_{1}\right)$,

$$
\operatorname{det}(\boldsymbol{Q}(s)-\boldsymbol{Q}(t))=\frac{\theta(t-s)}{\theta(t) \theta(s)}
$$

The restrictions imposed on the function $\theta(t)$ by (2) are quite strong, though implicit. For instance, if for a given exponential polynomial $\theta(t)$ the equation (2) has a unique solution $\boldsymbol{Q}(t)$, then the harmonic space with the density function $\theta(t)$ is two-point homogeneous (if it exists):

Proposition 3. Let $M^{n}$ be a harmonic manifold with the volume density function $\theta(t)$. Suppose that the equation (2) for a symmetric operator function $\boldsymbol{Q}(t): \mathbb{R}^{n-1} \rightarrow$

$\mathbb{R}^{n-1}$ with an asymptotic expansion $t^{-1} \mathrm{id}_{n-1}+O(t)$ at 0 has a unique solution up to a conjugation by a constant orthogonal transformation. Then $M^{n}$ is two-point homogeneous.

The paper is organized as follows. In Section 2 we give the proof of Proposition 1 using technical Proposition 4 (moved to Section 4) on the structure of algebraic curvature tensors satisfying (1). The proof of Theorem 2 and Propositions 2 and 3 are contained in Section 3.

The author is thankful to Prof. L. Vanhecke and to Prof. A. Ranjan for many useful comments and references.

\section{Five-dimensional harmonic spaces. Proof of Proposition 1}

We start with an algebraic description of the curvature tensor of a Riemannian manifold $M^{5}$ satisfying the first two Ledger formulae (1).

An algebraic curvature tensor in a Euclidean space is a $(3,1)$ tensor having the same symmetries as the curvature tensor of a Riemannian manifold. Given an or- 
thonormal basis $\left\{e_{i}\right\}$, denote $R_{i j k l}=\left\langle R\left(e_{i}, e_{j}\right) e_{k}, e_{l}\right\rangle$ the components of the algebraic curvature tensor $R$, and let $\kappa_{i j}=R_{i j i j}$.

Proposition 4. Let $R$ be an algebraic curvature tensor in $\mathbb{R}^{5}$ satisfying (1). Then there exists an orthonormal basis $\left\{e_{i}\right\}$ such that

$$
\begin{gathered}
\kappa_{12}=\kappa_{13}=\kappa_{23}=\kappa_{24}=\kappa_{34}=\alpha-\gamma, \\
\kappa_{25}=\kappa_{35}=\alpha-3 \gamma, \quad \kappa_{15}=\kappa_{45}=\alpha, \quad \kappa_{14}=\alpha-4 \gamma, \\
R_{1234}=\gamma, \quad R_{1235}=\sqrt{3} \gamma, \quad R_{1324}=-\gamma, \quad R_{1325}=\sqrt{3} \gamma \\
R_{1423}=-2 \gamma, \quad R_{2425}=\sqrt{3} \gamma, \quad R_{3435}=-\sqrt{3} \gamma,
\end{gathered}
$$

and all the other components of $R$ vanish.

The proof of Proposition 4 (which is somewhat technically involved) is moved to Section 4. In this section, we prove Proposition 1 assuming Proposition 4.

Let $M^{5}$ be a Riemannian manifold with the curvature tensor given by (3). Then $C=4 \alpha-6 \gamma, H=\left(\frac{C}{2}\right)^{2}+9 \gamma^{2}$, and so $\alpha$ and $\gamma$ must be constant on $M^{5}$. If $\gamma=0$, then the sectional curvature of $M^{5}$ is constant as follows from (3).

Assume that $\gamma \neq 0$. We want to show that $M^{5}$ is locally homothetic to $\mathrm{SU}(3) / \mathrm{SO}(3)$ or to $\mathrm{SL}(3) / \mathrm{SO}(3)$. To do that, we first prove that $M^{5}$ is locally symmetric using the second Bianchi identity, and then compare its curvature tensor with that of $\mathrm{SL}(3) / \mathrm{SO}(3)$.

Let $\omega^{i}$ be the 1 -forms dual to $e_{i}$, and let $\psi_{i}^{j}, \Omega_{i}^{j}$ be the connection and the curvature forms, respectively:

$$
\begin{aligned}
& d \omega^{i}=-\psi_{j}^{i} \wedge \omega^{j}, \quad d \psi_{j}^{i}=-\psi_{k}^{i} \wedge \psi_{j}^{k}+\Omega_{j}^{i}, \\
& \psi_{j}^{i}+\psi_{i}^{j}=0, \quad \Omega_{j}^{i}=-\Omega_{i}^{j}=\frac{1}{2} R_{i j k l} \omega^{k} \wedge \omega^{l} .
\end{aligned}
$$

Introduce the 2-forms

$$
\begin{gathered}
\Phi=-\omega^{1} \wedge \omega^{2}+\omega^{3} \wedge \omega^{4}+\sqrt{3} \omega^{3} \wedge \omega^{5} \\
\Psi=\omega^{2} \wedge \omega^{4}+\omega^{1} \wedge \omega^{3}-\sqrt{3} \omega^{2} \wedge \omega^{5}, \\
\Gamma=4 \omega^{1} \wedge \omega^{4}+2 \omega^{2} \wedge \omega^{3}
\end{gathered}
$$

and let $\Xi_{j}^{i}=\frac{1}{\gamma}\left(\Omega_{j}^{i}-\alpha \omega^{i} \wedge \omega^{j}\right)$. Since $\alpha$ and $\gamma$ are constant, the second Bianchi identity $d \Omega_{j}^{i}=\Omega_{k}^{i} \wedge \psi_{j}^{k}-\Omega_{j}^{k} \wedge \psi_{k}^{i}$ implies

$$
d \Xi_{j}^{i}=\Xi_{k}^{i} \wedge \psi_{j}^{k}-\Xi_{j}^{k} \wedge \psi_{k}^{i}
$$


The 2-forms $\Xi_{j}^{i}$ can be found from (3):

$$
\begin{array}{lll}
\Xi_{2}^{1}=\Phi, & \Xi_{4}^{3}=-\Phi, & \Xi_{5}^{3}=-\sqrt{3} \Phi, \\
\Xi_{3}^{1}=-\Psi, & \Xi_{4}^{2}=-\Psi, & \Xi_{5}^{2}=\sqrt{3} \Psi, \\
\Xi_{4}^{1}=-\Gamma, & \Xi_{3}^{2}=-\frac{1}{2} \Gamma, & \Xi_{5}^{1}=\Xi_{5}^{4}=0 .
\end{array}
$$

Differentiating $\Xi_{5}^{1}=\Xi_{5}^{4}=\Xi_{2}^{1}+\Xi_{4}^{3}=\Xi_{3}^{1}-\Xi_{4}^{2}=4 \Xi_{2}^{1}+\Xi_{4}^{3}+\sqrt{3} \Xi_{5}^{3}=0$ we get, respectively:

$$
\begin{aligned}
\Phi \wedge\left(\psi_{5}^{2}+\sqrt{3} \psi_{3}^{1}\right)-\Psi \wedge\left(\psi_{5}^{3}+\sqrt{3} \psi_{2}^{1}\right)-\Gamma \wedge \psi_{5}^{4}=0, \\
\Phi \wedge\left(\psi_{5}^{3}-\sqrt{3} \psi_{4}^{3}\right)+\Psi \wedge\left(\psi_{5}^{2}+\sqrt{3} \psi_{4}^{2}\right)+\Gamma \wedge \psi_{5}^{1}=0, \\
\Phi \wedge \psi_{5}^{4}+\Psi \wedge \psi_{5}^{1}+\frac{\sqrt{3}}{2} \Gamma \wedge\left(\psi_{4}^{2}-\psi_{3}^{1}\right)=0, \\
-\Phi \wedge \psi_{5}^{1}+\Psi \wedge \psi_{5}^{4}+\frac{\sqrt{3}}{2} \Gamma \wedge\left(\psi_{2}^{1}+\psi_{4}^{3}\right)=0, \\
\Psi \wedge\left(6 \psi_{3}^{2}-3 \psi_{4}^{1}+5 \sqrt{3} \psi_{5}^{1}\right)+\Gamma \wedge\left(\frac{9}{2} \psi_{4}^{2}-3 \psi_{3}^{1}+\frac{\sqrt{3}}{2} \psi_{5}^{2}\right)=0 .
\end{aligned}
$$

From (4) we obtain by a straightforward computation:

Lemma 1. If $\Phi \wedge x+\Psi \wedge y+\Gamma \wedge z=0$ for 1-forms $x, y$, $z$, then

$$
\begin{aligned}
& x=2 z_{3} \omega^{1}-z_{4} \omega^{2}+\left(2 z_{1}-y_{2}\right) \omega^{3}-2 z_{2} \omega^{4}-2 \sqrt{3} z_{2} \omega^{5}, \\
& y=2 z_{2} \omega^{1}+y_{2} \omega^{2}+z_{4} \omega^{3}+2 z_{3} \omega^{4}-2 \sqrt{3} z_{3} \omega^{5}, \\
& z=z_{1} \omega^{1}+z_{2} \omega^{2}+z_{3} \omega^{3}+z_{4} \omega^{4} .
\end{aligned}
$$

Equations $(6,7)$ and Lemma 1 imply that the $\omega^{5}$-components of $\psi_{5}^{1}$ and $\psi_{5}^{4}$ vanish. Applying this and Lemma 1 to $(8,9)$ we find:

$$
\begin{array}{cl}
\psi_{5}^{1}=a \omega^{2}-b \omega^{3}, & \psi_{5}^{4}=b \omega^{2}+a \omega^{3}, \\
\psi_{4}^{2}-\psi_{3}^{1}=\frac{2}{\sqrt{3}}\left(a \omega^{1}-b \omega^{4}\right), & \psi_{2}^{1}+\psi_{4}^{3}=\frac{2}{\sqrt{3}}\left(b \omega^{1}+a \omega^{4}\right) .
\end{array}
$$

Substituting $\psi_{5}^{1}$ and $\psi_{5}^{4}$ to $(6,7)$ and using Lemma 1 again we obtain

$$
\begin{aligned}
& \psi_{5}^{2}+\sqrt{3} \psi_{3}^{1}=-2 a \omega^{1}+c_{1} \omega^{3}+2 b \omega^{4}+2 \sqrt{3} b \omega^{5}, \\
& \psi_{5}^{3}+\sqrt{3} \psi_{2}^{1}=2 b \omega^{1}+c_{1} \omega^{2}+2 a \omega^{4}-2 \sqrt{3} a \omega^{5}, \\
& \psi_{5}^{3}-\sqrt{3} \psi_{4}^{3}=-2 b \omega^{1}+c_{2} \omega^{3}-2 a \omega^{4}-2 \sqrt{3} a \omega^{5}, \\
& \psi_{5}^{2}+\sqrt{3} \psi_{4}^{2}=2 a \omega^{1}-c_{2} \omega^{2}-2 b \omega^{4}+2 \sqrt{3} b \omega^{5} .
\end{aligned}
$$

Subtracting the third equation from the second one and substituting the expression for $\psi_{2}^{1}+\psi_{4}^{3}$ from (11) we find that $a=b=c_{1}=c_{2}=0$, and so

$$
\psi_{5}^{1}=\psi_{5}^{4}=\psi_{4}^{2}-\psi_{3}^{1}=\psi_{2}^{1}+\psi_{4}^{3}=\psi_{5}^{2}+\sqrt{3} \psi_{3}^{1}=\psi_{5}^{3}+\sqrt{3} \psi_{2}^{1}=0 .
$$


It now follows from (10) that $2 \psi_{3}^{2}=\psi_{4}^{1}$. Thus there exist 1 -forms $\tau, \eta, \sigma$ such that the connection forms are

$$
\begin{gathered}
\psi_{3}^{1}=\psi_{4}^{2}=\tau, \quad \psi_{5}^{2}=-\sqrt{3} \tau, \quad \psi_{2}^{1}=-\eta, \quad \psi_{4}^{3}=\eta, \quad \psi_{5}^{3}=\sqrt{3} \eta, \\
\psi_{3}^{2}=\sigma, \quad \psi_{4}^{1}=2 \sigma, \quad \psi_{5}^{1}=\psi_{5}^{4}=0 .
\end{gathered}
$$

Then we have $0=d\left(\psi_{2}^{1}+\psi_{4}^{3}\right)=-\psi_{k}^{1} \wedge \psi_{2}^{k}+\Omega_{2}^{1}-\psi_{k}^{3} \wedge \psi_{4}^{k}+\Omega_{4}^{3}=\Omega_{2}^{1}+\Omega_{4}^{3}=$ $\gamma\left(\Xi_{2}^{1}+\Xi_{4}^{3}\right)+\alpha\left(\omega^{1} \wedge \omega^{2}+\omega^{3} \wedge \omega^{4}\right)$, and so $\alpha=0$ by (5).

Then by (3) the nonzero components of the curvature tensor are

$$
\begin{array}{ll}
R_{1212}=-\gamma, \quad R_{1234}=\gamma, \quad R_{1235}=\sqrt{3} \gamma, R_{1324}=-\gamma, & R_{1313}=-\gamma, \\
R_{1325}=\sqrt{3} \gamma, R_{1414}=-4 \gamma, R_{1423}=-2 \gamma, R_{2323}=-\gamma, & R_{2424}=-\gamma, \\
R_{2425}=\sqrt{3} \gamma, R_{2525}=-3 \gamma, R_{3434}=-\gamma, & R_{3435}=-\sqrt{3} \gamma, R_{3535}=-3 \gamma .
\end{array}
$$

Using $(12,13)$ we find that the covariant derivative of $R$ given by

$$
R_{i j k l, h} \omega^{h}=d R_{i j k l}-R_{i j k s} \psi_{l}^{s}-R_{i j s l} \psi_{k}^{s}-R_{i s k l} \psi_{j}^{s}-R_{s j k l} \psi_{i}^{s}
$$

vanishes, and so the Riemannian space $M^{5}$ is locally symmetric.

Consulting the list of symmetric spaces [8] we find that the only possible candidates for $M^{5}$ are $\mathrm{SU}(3) / \mathrm{SO}(3)$, or its noncompact dual SL(3)/ $\mathrm{SO}(3)$. This is indeed the case: the tangent space $\mathfrak{m}$ to $\mathrm{SL}(3) / \mathrm{SO}(3)$ at the origin can be identified with a Lie triple system of $3 \times 3$ real traceless symmetric matrices. The inner product and the curvature tensor are given by

$$
\langle X, Y\rangle=\operatorname{Tr}(X Y), \quad R(X, Y) Z=-[[X, Y], Z], \quad X, Y, Z \in \mathfrak{m} .
$$

Then, with respect to the orthonormal basis

$$
\begin{gathered}
e_{1}=\frac{1}{\sqrt{2}}\left(\begin{array}{lll}
0 & 1 & 0 \\
1 & 0 & 0 \\
0 & 0 & 0
\end{array}\right) \quad e_{2}=\frac{1}{\sqrt{2}}\left(\begin{array}{lll}
0 & 0 & 1 \\
0 & 0 & 0 \\
1 & 0 & 0
\end{array}\right) \quad e_{3}=\frac{1}{\sqrt{2}}\left(\begin{array}{lll}
0 & 0 & 0 \\
0 & 0 & 1 \\
0 & 1 & 0
\end{array}\right) \\
e_{4}=\frac{1}{\sqrt{2}}\left(\begin{array}{ccc}
-1 & 0 & 0 \\
0 & 1 & 0 \\
0 & 0 & 0
\end{array}\right) \quad e_{5}=\frac{1}{\sqrt{6}}\left(\begin{array}{ccc}
1 & 0 & 0 \\
0 & 1 & 0 \\
0 & 0 & -2
\end{array}\right)
\end{gathered}
$$

the components of the curvature tensor of $\mathrm{SL}(3) / \mathrm{SO}(3)$ are proportional to those given by (13).

\section{The volume density function and the matrix equation}

In this section, we prove Theorem 2 and Propositions 2 and 3. 
Let $\gamma=\gamma(t)$ be a geodesic on a Riemannian manifold $M^{n}$, with $t$ an arclength parameter, $\gamma(0)=x, \dot{\gamma}(0)=T \in T_{x} M^{n}$. Let $\gamma\left(t_{1}\right), t_{1}>0$ be the first point conjugate to $x$ along $\gamma$.

For every $t \in\left(0, t_{1}\right)$ we define the operator $Q(t): L \rightarrow L, L=T^{\perp}$ putting $\boldsymbol{Q}(t) X=Z$, if $J_{0, Z}(t)=J_{X, 0}(t)$, where $J_{X, Y}$ is the Jacobi field along $\gamma$ such that $J_{X, Y}(0)=X, J_{X, Y}^{\prime}(0)=Y$, prime stands for the covariant derivative along $\gamma$.

The operator $\boldsymbol{Q}(t)$ is symmetric. Indeed, for $t_{0} \in\left(0, t_{1}\right)$ and $X, Y \in L$, let $J_{1}=J_{0, \boldsymbol{Q}\left(t_{0}\right) X}, J_{2}=J_{X, 0}, J_{3}=J_{0, \boldsymbol{Q}\left(t_{0}\right) Y}, J_{4}=J_{Y, 0}$ be the corresponding Jacobi fields. Denote $U=J_{1}\left(t_{0}\right)=J_{2}\left(t_{0}\right), V=J_{3}\left(t_{0}\right)=J_{4}\left(t_{0}\right)$. Since for any two Jacobi fields $I$ and $J$ along a geodesic, the function $\left\langle I^{\prime}, J\right\rangle-\left\langle I, J^{\prime}\right\rangle$ is constant, we obtain at the point $t_{0}$ :

$$
\begin{gathered}
\left\langle J_{1}^{\prime}, V\right\rangle-\left\langle J_{3}^{\prime}, U\right\rangle=\left\langle J_{2}^{\prime}, V\right\rangle-\left\langle J_{4}^{\prime}, U\right\rangle=0, \\
\left\langle J_{1}^{\prime}, V\right\rangle-\left\langle J_{4}^{\prime}, U\right\rangle=\left\langle\boldsymbol{Q}\left(t_{0}\right) X, Y\right\rangle, \quad\left\langle J_{2}^{\prime}, V\right\rangle-\left\langle J_{3}^{\prime}, U\right\rangle=-\left\langle\boldsymbol{Q}\left(t_{0}\right) Y, X\right\rangle,
\end{gathered}
$$

and the claim follows.

Fix an orientation on a neighbourhood of $\gamma_{\mid}\left(0, t_{1}\right)$. Choose orthonormal vector fields $\left\{e_{1}, \ldots, e_{n-1}, e_{n}=\dot{\gamma}(t)\right\}$ parallel along $\gamma$ and forming a positively oriented basis. Denote $R(t)=\left(R_{i j}(t)\right), i, j=1, \ldots, n-1$ the matrix of the Jacobi operator with respect to the basis $\left\{e_{1}, \ldots, e_{n-1}\right\}$. Let $A_{s}(t)$ be an $(n-1) \times(n-1)$-matrix satisfying the Jacobi equation $\ddot{A}_{s}(t)+R(t) A_{s}(t)=0$ along $\gamma$, with the initial conditions $A_{s}(s)=0, \dot{A}_{s}(s)=I_{n-1}$, the identity matrix, and ${ }^{\circ}=d / d t$. Denote $\theta_{s}(t)=$ $\operatorname{det} A_{s}(t), s, t \in \mathbb{R}$.

If the space $M^{n}$ is harmonic, then for any choice of $\gamma$ and $s, \theta_{s}(t)=\theta(t-s)$ [2, Ch. 6]. The volume density function $\theta(t)$ is analytic and $\theta(-t)=(-1)^{n-1} \theta(t)$.

Proof of Proposition 2. Let $A(t), B(t)$ be two matrix solutions of the Jacobi equation such that $A(0)=\dot{B}(0)=0, \dot{A}(0)=B(0)=I_{n}$. Then the matrix of the operator $\boldsymbol{Q}(t)$ is $Q(t)=A^{-1}(t) B(t)$, and we have $A_{s}(t)=A(t)(Q(s)-Q(t)) M(s)$, where $M(s)=$ $\left(\dot{A}(s) A^{-1}(s) B(s)-\dot{B}(s)\right)^{-1}$, for $s, t \in\left(0, t_{1}\right)$. Indeed, the matrix $A(t)(Q(s)-$ $Q(t)) M(s)=A(t)(Q(s) M(s))-B(t) M(s)$ satisfies the Jacobi equation $\frac{d^{2}}{d t^{2}} A_{s}(t)+$ $R(t) A_{s}(t)=0$, it vanishes at $t=s$, and $\frac{d}{d t}(A(t)(Q(s)-Q(t)) M(s))_{\mid t=s}=I_{n-1}$. We have $\operatorname{det}\left(M A^{-1 t}\right)=\operatorname{det}\left(A^{t} \dot{A} A^{-1} B-A^{t} \dot{B}\right)^{-1}=1$, since $A^{t} \dot{A}-\dot{A}^{t} A=0$ and $A^{t} \dot{B}-\dot{A}^{t} B=-I_{n}$. Hence det $M(s)=\theta(s)$, and so $\theta(s-t)=\operatorname{det} A_{s}(t)=$ $\theta(t) \theta(s) \operatorname{det}(Q(s)-Q(t))$.

Remark 1. We can give another interpretation to (2). Denote $P_{t 0}: T_{\gamma(t)} M^{n} \rightarrow$ $T_{x} M^{n}$ the parallel translation along $\gamma$, and for every $t \in \mathbb{R}$ define a linear map $F(t): L \oplus L \rightarrow L$ by $F(t)(X, Y)=P_{t 0} J_{X, Y}(t)$. Denote $\omega(t)$ a volume form on $F^{*}(t)\left(L^{*}\right)$. Then $\omega(t)$ is a curve on the Grassmannian $G(n-1,2 n-2) \subset \Lambda^{n}(L \oplus L)^{*}$, and (2) has the form:

$$
\omega(s) \wedge \omega(t)=\theta(t-s) * 1
$$


for all $t, s \in \mathbb{R}$. The proof follows from the fact that

$$
\operatorname{det}\left(\begin{array}{ll}
A(s) & B(s) \\
A(t) & B(t)
\end{array}\right)=\operatorname{det} A(s) \operatorname{det} A(t) \operatorname{det}(Q(s)-Q(t))=\theta(t-s),
$$

by (2), with $A$ and $B$ as in the proof of Proposition 2.

One might compare (14) to the fact that for a Nice Embedding $\Phi$ of a harmonic manifold [2], [13], [14], the equation $\langle\Phi(\gamma(t)), \Phi(\gamma(s))\rangle=\Theta(t-s)$ holds, with some function $\Theta$, along every geodesic $\gamma$.

Proof of Theorem 2. Let $t, s \in\left(0, t_{1}\right)$. Multiplying (2) by $\theta(t) \theta(s)$ and expanding the determinant on the left hand side, we obtain $\theta(t-s)=\sum_{\alpha} f_{\alpha}(t) g_{\alpha}(s)$, with functions $f_{\alpha}, g_{\alpha}$ being linear combinations of minors of the matrix $Q$ multiplied by $\theta$. Taking appropriate linear combinations, we can assume that both sets of functions $\left\{f_{\alpha}\right\}$ and $\left\{g_{\alpha}\right\}$ are linearly independent over $\mathbb{R}$. Let $N=\operatorname{rk}\left\{f_{\alpha}\right\}=\operatorname{rk}\left\{g_{\alpha}\right\}$. We have $0=(\partial / \partial t+\partial / \partial s) \theta(t-s)=\sum_{\alpha}\left(\dot{f}_{\alpha}(t) g_{\alpha}(s)+f_{\alpha}(t) \dot{g}_{\alpha}(s)\right)$. So there exists an $N \times N$ constant matrix $C$ such that $\dot{f}=C f, \dot{g}=-C^{t} g$, where $f=\left(f_{1}, \ldots, f_{N}\right)^{t}$, $g=\left(g_{1}, \ldots, g_{N}\right)^{t}$. Thus $f(t)=e^{C t} u, g(s)=\left(e^{-C s}\right)^{t} v$ for some constant vectors $u, v \in \mathbb{R}^{N}$. Then $\theta(t-s)=\langle f(t), g(s)\rangle=\left\langle e^{C t} u,\left(e^{-C s}\right)^{t} v\right\rangle=\left\langle e^{C(t-s)} u, v\right\rangle$, that is, $\theta(x)=\left\langle e^{C x} u, v\right\rangle$, with some constant matrix $C$ and constant vectors $u, v$.

Remark 2. As it follows from the proof, the number of monomials of the exponential polynomial $\theta$ is not greater than $\left(\left(\begin{array}{c}2 n \\ n\end{array}\right)+2^{n}\right) / 2$.

Proof of Proposition 3. Let $\gamma(t), \tilde{\gamma}(t)$ be two geodesics on $M^{n}$. We equip all the objects related to $\tilde{\gamma}(t)$ with the tilde. Construct the operators $\boldsymbol{Q}(t), \tilde{\boldsymbol{Q}}(t)$ for $\gamma(t)$ and $\tilde{\gamma}(t)$ respectively. By assumption, we can choose orthonormal bases at $T_{\gamma(0)} M^{n}$ and $T_{\tilde{\gamma}(0)} M^{n}$ such that $Q(t)=\tilde{Q}(t)$.

Introduce the matrices $A$ and $B$ as in the proof of Proposition 2. Then $Q=A^{-1} B$ and $B^{t} \dot{B}-\dot{B}^{t} B=0, A^{t} \dot{B}-\dot{A}^{t} B=-I$, and a direct computation shows that $\left(Q^{-1}(t)\right)^{\cdot}=\left(B(t)^{t} B(t)\right)^{-1}$.

It follows that $\tilde{B}(t)=V(t) B(t)$ with $V(t)$ an orthogonal matrix function. Since $\tilde{B}^{t} \dot{\tilde{B}}-\dot{\tilde{B}}^{t} \tilde{B}=B^{t} \dot{B}-\dot{B}^{t} B=0$, the matrix $V(t)$ must be constant. Thus $\tilde{R}(t)=$ $V R(t) V^{-1}$ for some constant orthogonal matrix $V$.

So, for any two geodesics $\gamma(t)$ and $\tilde{\gamma}(t)$, we can choose parallel orthonormal bases such that $\tilde{R}(t)=R(t)$. In particular, for any point $x \in M^{n}$ and unit vectors $X, Y \in T_{x} M^{n}$, the operators $\left(\left(\nabla_{X} R\right)(X, .) X\right)_{\mid X^{\perp}}$ and $\left(\left(\nabla_{Y} R\right)(Y, .) Y\right)_{\mid Y^{\perp}}$ are equal, up to an orthogonal conjugation. By [14, Lemma 1.1] applied to the operator $\left(\left(\nabla_{X} R\right)(X, .) X\right)_{\mid X^{\perp}}$, we get $\nabla R=0$ and the claim follows.

Example 1. If $M^{n}$ is two-point homogeneous, then the operator $\boldsymbol{Q}(t)$ is diagonalizable, with diagonal entries of the form $\lambda \cot (\lambda t), \lambda \operatorname{coth}(\lambda t)$ or $t^{-1}$ depending on the curvature. 
Example 2. In this example, we use the results and notation of [1, Ch. 4].

Let $M^{4 n+3}=\mathfrak{n} \oplus \mathfrak{z} \oplus \mathfrak{a}$ be a Damek-Ricci space with $\operatorname{dim} \mathfrak{z}=2$, and let $\gamma(t)$ be a generic geodesic starting at the origin. Identifying tangent spaces $T_{\gamma(t)} M$ along $\gamma(t)$ via left translation, we have $\dot{\gamma}(t)=V(t)+Y(t)+s(t) A$, with $V=V(0), Y(0)=Y$, $s(0)=s$ nonzero, and $T_{\gamma(t)} M=\mathfrak{s}_{4} \oplus \mathfrak{p} \oplus \mathfrak{q}$. This decomposition is orthogonal, with subspaces $\mathfrak{s}_{4}, \mathfrak{p}$ and $\mathfrak{q}$ being $R(t)$-invariant and parallel along $\gamma$, since $\dot{\gamma}(t) \in \mathfrak{s}_{4}$ and the two-dimensional subspace $\mathfrak{b}=\operatorname{Span}\left(V(t), J_{Y(t)} V(t)\right)$ does not depend on $t$.

Now $\mathfrak{s}_{4}$ is tangent to a totally geodesic $\mathbb{C} H^{2} \subset M$, and $R(t)_{\mid \mathfrak{p}}=-\frac{1}{4} \mathrm{id}_{\mid \mathfrak{p}}$. Hence $\boldsymbol{Q}(t)$ is diagonalizable on the $4 n-1$-dimensional space $\left(\mathfrak{s}_{4} \oplus \mathfrak{p}\right) \cap \dot{\gamma}(t)^{\perp}$, with diagonal entries $\operatorname{coth}(t)$ of multiplicity one and $\frac{1}{2} \operatorname{coth}(t / 2)$ of multiplicity $4 n-2$.

The behavior of $\boldsymbol{Q}(t)$ is more complicated on the three-dimensional space $\mathfrak{q}$. First find six Jacobi fields along $\gamma$ lying in $\mathfrak{q}$. Denote $\alpha=s+i\|Y\|$ and introduce a complex function $\phi(t)=\alpha \sinh (t / 2)-\cosh (t / 2)$ and a real function $f(t)=\phi \bar{\phi}=$ $(s \sinh (t / 2)-\cosh (t / 2))^{2}+\|Y\|^{2} \sinh ^{2}(t / 2)$ (note that $f(t)=1 / h(t)$, the function $h(t)$ defined in 4.1 .11 of [1]). Let $X \in \mathfrak{z}$ be a unit vector orthogonal to $Y$. Then the vector field $f(t) X$ is Jacobi, which can be checked directly.

Taking this into account we can rewrite the Jacobi equation for a vector field $U(t)+g(t) X \subset \mathfrak{q}$ as follows:

$$
\begin{gathered}
\ddot{U}-J_{Y(t)} \dot{U}+\frac{\dot{f}}{2 f} J_{Y(t)} U-\frac{1}{4}\left(1+\|Y(t)\|^{2}\right) U+\frac{c}{f} J_{X} V(t)=0, \\
g \dot{f}-f \dot{g}+f\left\langle J_{X} U, V(t)\right\rangle=c
\end{gathered}
$$

for some constant $c$. Denote $W(t)=J_{X} U(t), J=\|Y(t)\|^{-1} J_{Y(t)}$. Then $W(t)$ is in $\mathfrak{b}$, and $J: \mathfrak{b} \rightarrow \mathfrak{b}$ is a skew-symmetric orthogonal operator. Introduce complex valued functions $w(t)=(\langle W(t), V\rangle+i\langle W(t), J V\rangle) /\|V\|, v(t)=(\langle V(t), V\rangle+$ $i\langle V(t), J V\rangle) /\|V\|$. The Jacobi equation now has the form

$$
\begin{gathered}
\ddot{w}-i\|Y\| f^{-1} \dot{w}-\frac{1}{4}\left(1+\|Y\|^{2} f^{-2}-2 i\|Y\| \dot{f} f^{-2}\right) w-c f^{-1} v=0, \\
g \dot{f}-f \dot{g}+f \operatorname{Re}(w \bar{v})=c,
\end{gathered}
$$

and its general solution is given by

$$
\begin{aligned}
w= & f^{-1 / 2}\left(A \phi^{2}+B \bar{\phi}^{2}+C \phi \bar{\phi}\right), \\
g= & 2 A\|V\|^{-1} \cosh (t / 2) \operatorname{Re}\left(\left(\alpha-\alpha^{-1}\right) \phi\right)-2\|V\| \cosh (t / 2) \operatorname{Re}(C \bar{\phi} / \alpha) \\
& -2\|V\| f \cosh (t / 2)\left(\left(1-\|V\|^{2}\right) \sinh (t / 2)-s \cosh (t / 2)\right) \operatorname{Re}\left(B \phi^{-2} \alpha^{-2}\right),
\end{aligned}
$$

with constants $A \in \mathbb{R}, B, C \in \mathbb{C}$ and $c=-2 A\|Y\|^{2} /\|V\|$. This gives explicit formulae for Jacobi fields.

The matrix of the operator $Q(t)_{\mid \mathfrak{q}}$ in the orthonormal basis $e_{1}=J_{X} V /\|V\|$, $e_{2}=-J_{X} J V /\|V\|, e_{3}=X$ has the form $f(t)^{-3}\left(V(t)^{t} \hat{Q}(t) V(t)\right)$, where $\hat{Q}(t)$ is a 
$3 \times 3$ symmetric matrix with entries

$$
\begin{gathered}
\hat{Q}_{11}=\left(\|V\|^{2} \tanh (t / 2)+\operatorname{coth}(t / 2)\right) / 2, \quad \hat{Q}_{12}=0, \quad \hat{Q}_{22}=2 \operatorname{coth}(t / 2), \\
\hat{Q}_{13}=-\|V\| \tanh (t / 2)\left(\left(1-\|V\|^{2}\right) \sinh (t / 2)-s \cosh (t / 2)\right), \\
\hat{Q}_{23}=\|Y\|\|V\| \sinh (t / 2) / 2, \quad \hat{Q}_{33}=f(t)\left(\left(1-\|V\|^{2}\right) \tanh (t / 2)+\operatorname{coth}(t / 2)\right) / 2,
\end{gathered}
$$

and

$$
V(t)=\left(\begin{array}{ccc}
s \sinh (t / 2)-\cosh (t / 2) & \|Y\| \sinh (t / 2) & 0 \\
-\|Y\| \sinh (t / 2) & s \sinh (t / 2)-\cosh (t / 2) & 0 \\
0 & 0 & 1
\end{array}\right) .
$$

So the matrix equation (2), with the function $\theta(t)=4 \sinh ^{2}(t / 2) \sinh (t)$, has a continuous family of solutions of the form given above.

\section{Proof of Proposition 4}

We prove Proposition 4 by explicitly solving the equations (1), the first two Ledger conditions. First, in Lemma 2, we construct a specific orthonormal basis for $\mathbb{R}^{5}$, in which the algebraic curvature tensor $R$ has a simple structure. Then, with some computations, we find that $R$ have the required form (3). Note that the constant curvature tensor is a particular case of (3), when $\gamma=0$.

For an orthonormal basis $\left\{e_{i}\right\}$ for $\mathbb{R}^{5}$, denote $R_{i j k l}=\left\langle R\left(e_{i}, e_{j}\right) e_{k}, e_{l}\right\rangle$ the components of the algebraic curvature tensor. Let $\kappa(\sigma)$ be the sectional curvature of a two-plane $\sigma$, in particular, denote $\kappa_{i j}=\kappa_{j i}=R_{i j i j}$ the sectional curvature of the two-plane spanned by vectors $e_{i}, e_{j}$. It will be convenient to set $\kappa_{i i}=0$.

The equations (1) have the following form [2, equation (6.50)]:

$$
\begin{gathered}
\operatorname{Ric}_{i j}=\sum_{p} R_{i p j p}=C \delta_{i j}, \quad 1 \leq i, j \leq 5 \\
\operatorname{Sym}_{i j k l}\left(\sum_{p q} R_{i p j q} R_{k p l q}\right)=H \operatorname{Sym}_{i j k l}\left(\delta_{i j} \delta_{k l}\right), \quad 1 \leq i, j, k, l \leq 5,
\end{gathered}
$$

where $\delta_{i j}$ is the Kronecker delta, all the summations are from 1 to 5, and Sym denotes the sum by all permutations of the subscripts $i, j, k, l$. Expanding the equation (16) we find

$$
\begin{gathered}
\sum_{p q} R_{i p i q}^{2}=H, \\
\sum_{p q} R_{i p i q} R_{i p j q}=0, \\
\sum_{p q}\left(R_{i p i q} R_{j p j q}+R_{i p j q}^{2}+R_{i p j q} R_{j p i q}\right)=H, \\
\sum_{p q}\left(R_{i p i q} R_{j p k q}+R_{i p j q} R_{i p k q}+R_{i p j q} R_{k p i q}\right)=0, \\
\operatorname{Sym}_{j k l}\left(\sum_{p q} R_{i p j q} R_{k p l q}\right)=0,
\end{gathered}
$$


where $i, j, k, l$ are pairwise nonequal.

We will use the following index convention throughout this section:

$$
1 \leq i, j, k, l, p, q \leq 5, \quad 2 \leq a, b, c, d, f, g \leq 5,
$$

unless the bounds are explicitly indicated.

The sectional curvature $\kappa=\kappa(\sigma)$ is a differentiable function on the Grassmannian $G(2,5)$ of two-planes in $\mathbb{R}^{5}$. We call a two-plane $\sigma \in \mathbb{R}^{5}$ critical if it is a critical point for $\kappa$. It is easy to see that $\sigma=\operatorname{Span}(U, V), U, V \in \mathbb{R}^{5}$ is critical if and only if $R(U, V, U, W)=R(U, V, V, W)=0$ for any $W \in \sigma^{\perp}$.

Lemma 2. There exists an orthonormal basis $\left\{e_{i}\right\}$ for $\mathbb{R}^{5}$ such that every two-plane $\operatorname{Span}\left(e_{1}, e_{a}\right)$ is critical, or equivalently

$$
R_{1 a 1 b}=R_{1 a b a}=0, \quad b \neq 1, a .
$$

Proof. To construct the required basis we take a critical two-plane $\sigma$ and choose an orthonormal basis $e_{1}, e_{2}$ in it. Then the subspace $L=\sigma^{\perp}$ is an invariant subspace of the Jacobi operator $R_{e_{1}}$. Choosing $e_{3}, e_{4}, e_{5}$ to be orthonormal eigenvectors of the restriction of $R_{e_{1}}$ to $L$ we obtain

$$
R_{121 a}=R_{12 a 2}=R_{1 a 1 b}=0, \quad 3 \leq a \neq b \leq 5 .
$$

Then using $(15,16)$ we show that the basis $e_{1}, e_{2}$ in $\sigma$ can be chosen in such a way that all the remaining components $R_{1 a b a}=0, a \neq b$ also vanish, so that (22) is satisfied.

From (15) $\operatorname{Ric}_{1 a}=\operatorname{Ric}_{2 a}=0$ for $a=3,4,5$, and $\operatorname{Ric}_{12}=0$. Using (23) we find

$$
\begin{gathered}
R_{1434}+R_{1535}=R_{1343}+R_{1545}=R_{1353}+R_{1454}=0, \\
R_{2434}+R_{2535}=R_{2343}+R_{2545}=R_{2353}+R_{2454}=0, \\
R_{1323}+R_{1424}+R_{1525}=0 .
\end{gathered}
$$

Let $\lambda_{1}, \lambda_{2}, \lambda_{3}$ be the eigenvalues of $R_{e_{1} \mid L}$ corresponding to the eigenvectors $e_{3}$, $e_{4}, e_{5}$, respectively. Then $R_{1 a 1 b}=\lambda_{a-2} \delta_{a b}, a, b=3,4,5$, and (18) with $i=1$, $j=3,4,5,2$ gives, respectively,

$$
\begin{gathered}
\lambda_{2} R_{1434}+\lambda_{3} R_{1535}=\lambda_{1} R_{1343}+\lambda_{3} R_{1545}=\lambda_{1} R_{1353}+\lambda_{2} R_{1454}=0, \\
\lambda_{1} R_{1323}+\lambda_{2} R_{1424}+\lambda_{3} R_{1525}=0 .
\end{gathered}
$$

The equations $(23,24,25)$ do not yet imply $(22)$ : we need to choose a specific basis $e_{1}, e_{2}$ in $\sigma$.

For a fixed orthonormal basis $E_{1}, E_{2}$ in $\sigma$, let $X(\phi)=\cos \phi E_{1}+\sin \phi E_{2}$, $\phi \in[0,2 \pi)$. Since the two-plane $\sigma$ is critical, both $X(\phi)$ and $X(\phi+\pi / 2)$ are 
eigenvectors of the Jacobi operator $R_{X(\phi)}$, with eigenvalues 0 and $\kappa(\sigma)$, respectively. Therefore, their span $\sigma$ and its orthogonal complement $L$ are invariant subspaces of $R_{X(\phi)}$, for any $\phi$. Hence we can define a symmetric operator $M(\phi): L \rightarrow L$, the restriction of $R_{X(\phi)}$ to $L$. It then follows from (1) that for all $\phi \in[0,2 \pi)$

$$
\operatorname{Tr} M(\phi)=\tilde{C}=C-\kappa(\sigma), \quad \operatorname{Tr} M^{2}(\phi)=\tilde{H}=H-\kappa(\sigma)^{2} .
$$

Explicitly, for $U \in L$

$$
\begin{aligned}
M(\phi) U= & \cos ^{2} \phi R_{E_{1}} U+\sin ^{2} \phi R_{E_{2}} U \\
& +\cos \phi \sin \phi\left(R\left(E_{1}, U\right) E_{2}+R\left(E_{2}, U\right) E_{1}\right) .
\end{aligned}
$$

We have several cases depending on the eigenvalues of $M(\phi)$.

Case 1. There exists $\phi$ such that all the eigenvalues of $M(\phi)$ are equal. Then by (26), $\tilde{C}^{2}=3 \tilde{H}$ and so the operator $M(\phi)$ is scalar: $M(\phi)=\frac{1}{3} \tilde{C} \mathrm{id}_{L}$. By (27), for all $U \in L, R\left(E_{1}, U, E_{2}, U\right)=0$, since $R_{E_{1}} U=M(0) U=\frac{1}{3} \tilde{C} U$ and $R_{E_{2}} U=$ $M(\pi / 2) U=\frac{1}{3} \tilde{C} U$. It follows that for $e_{1}=E_{1}, e_{2}=E_{2}$ and any orthonormal vectors $e_{3}, e_{4}, e_{5} \in L, R_{1 a 2 a}=0$ when $a=3,4,5$.

Introduce a linear operator $N: L \rightarrow L$ by $N U=R(Y, V) E_{1}$, where $Y, U, V \in L$ and $U=Y \times V$, the cross product in the three-dimensional space $L$. The operator $N$ is well-defined and symmetric. Indeed, let $Y$ and $Z$ be two orthonormal vectors in $L$ and $U=Y \times Z$. Then $Z=U \times Y, Y=Z \times U$ and so $\langle N Z, Y\rangle-\langle N Y, Z\rangle=$ $R\left(U, Y, E_{1}, Y\right)+R\left(U, Z, E_{1}, Z\right)=\operatorname{Ric}\left(U, E_{1}\right)=0$.

Let $e_{3}, e_{4}, e_{5} \in L$ be orthonormal eigenvectors of the operator $N$. Then $R_{1 a b a}=$ $\left\langle N\left(e_{b} \times e_{a}\right), e_{a}\right\rangle=0$ for all $3 \leq a \neq b \leq 5$.

Combining this with (23) we find that (22) is satisfied, hence all the two-planes $\operatorname{Span}\left(e_{1}, e_{a}\right)$ are critical.

Since the operator $M(\phi)$ is symmetric and analytic, its eigenvalues are analytic functions of $\phi$. If $\lambda(\phi)$ is an eigenvalue of $M(\phi)$, which is simple at $\phi=$ $\phi_{0}$, then the corresponding unit eigenvector $U(\phi)$ is also analytic in a neighbourhood of $\phi_{0}$, and $\lambda^{\prime}\left(\phi_{0}\right)=\frac{d}{d \phi}\langle M(\phi) U(\phi), U(\phi)\rangle_{\mid \phi=\phi_{0}}=\left\langle M^{\prime}\left(\phi_{0}\right) U\left(\phi_{0}\right), U\left(\phi_{0}\right)\right\rangle+$ $2 \lambda\left(\phi_{0}\right)\left\langle U^{\prime}\left(\phi_{0}\right), U\left(\phi_{0}\right)\right\rangle=\left\langle M^{\prime}\left(\phi_{0}\right) U\left(\phi_{0}\right), U\left(\phi_{0}\right)\right\rangle$.

We call $\phi_{0}$ a critical angle if it is critical for the function $\operatorname{det} M(\phi)$.

Modulo Case 1, one of the following two cases may occur.

Case 2. There exists a critical angle $\phi_{0}$ such that $\lambda_{1}\left(\phi_{0}\right), \lambda_{2}\left(\phi_{0}\right), \lambda_{3}\left(\phi_{0}\right)$ are pairwise nonequal. From (26) we have

$\left(\lambda_{1}(\phi)+\lambda_{2}(\phi)+\lambda_{3}(\phi)\right)^{\prime}=\left(\lambda_{1}^{2}(\phi)+\lambda_{2}^{2}(\phi)+\lambda_{3}^{2}(\phi)\right)^{\prime}=\left(\lambda_{1}(\phi) \lambda_{2}(\phi) \lambda_{3}(\phi)\right)_{\mid \phi=\phi_{0}}^{\prime}=0$.

This gives a system of linear equations for $\lambda_{i}^{\prime}\left(\phi_{0}\right)$, which implies $\lambda_{i}^{\prime}\left(\phi_{0}\right)=0$, since $\lambda_{i}\left(\phi_{0}\right)$ are pairwise nonequal. Then $\left\langle M^{\prime}\left(\phi_{0}\right) U_{i}, U_{i}\right\rangle=0$, where $U_{i}$ is a unit eigenvector of $M\left(\phi_{0}\right)$ corresponding to the eigenvalue $\lambda_{i}\left(\phi_{0}\right), i=1,2,3$. 
Define $e_{1}=X\left(\phi_{0}\right), e_{2}=X\left(\phi_{0}+\pi / 2\right)$ and $e_{i+2}=U_{i}, i=1,2,3$. Then by (27) $R_{1 a 2 a}=\frac{1}{2}\left\langle M^{\prime}\left(\phi_{0}\right) e_{a}, e_{a}\right\rangle=0$ for $a=3,4,5$.

Also, $R_{1434}=R_{1535}=R_{1545}=R_{1343}=R_{1353}=R_{1454}=0$ by $(24,25)$.

Thus all the equations (22) are satisfied.

Case 3. For any critical angle $\phi_{0}$, two of the three eigenvalues $\lambda_{i}\left(\phi_{0}\right)$ are equal. Let $\phi_{0}$ be a critical angle, and let the eigenvalues $\lambda_{i}=\lambda_{i}\left(\phi_{0}\right)$ be labelled in such a way that $\lambda_{1} \neq \lambda_{2}=\lambda_{3}$.

Choose $e_{1}=X\left(\phi_{0}\right), e_{2}=X\left(\phi_{0}+\pi / 2\right)$, and $e_{3}, e_{4}, e_{5}$ orthonormal eigenvectors of $M\left(\phi_{0}\right)$, with $e_{3}$ corresponding to $\lambda_{1}\left(e_{4}, e_{5}\right.$ can be chosen up to a rotation in the $\lambda_{2}$-eigenspace of $\left.M\left(\phi_{0}\right)\right)$.

As in Case 2, we find $\lambda_{1}^{\prime}\left(\phi_{0}\right)=0$. Then by (27) $R_{1323}=\frac{1}{2}\left\langle M^{\prime}\left(\phi_{0}\right) e_{3}, e_{3}\right\rangle=0$.

From the equations $(24,25)$ we obtain $R_{1343}=R_{1353}=R_{1545}=R_{1454}=0$. Combining this with (23) we find that the two-plane $\sigma^{\prime}=\operatorname{Span}\left(e_{1}, e_{3}\right)$ must be critical, while the two-planes $\operatorname{Span}\left(e_{1}, e_{4}\right)$ and $\operatorname{Span}\left(e_{1}, e_{5}\right)$ are critical if $R_{1424}=$ $R_{1434}=0$ and $R_{1525}=R_{1535}=0$, respectively.

From (26), $\lambda_{1}+2 \lambda_{2}=\tilde{C}, \lambda_{1}^{2}+2 \lambda_{2}^{2}=\tilde{H}$. So for every critical angle $\phi_{0}$, the eigenvalues of $M\left(\phi_{0}\right)$ are $\left\{\lambda_{1}^{\epsilon}, \lambda_{2}^{\epsilon}, \lambda_{2}^{\epsilon}\right\}, \epsilon= \pm$, where $\lambda_{1}^{ \pm}=\frac{1}{3}\left(\tilde{C} \mp \sqrt{6 \tilde{H}-2 \tilde{C}^{2}}\right)$, $\lambda_{2}^{ \pm}=\frac{1}{6}\left(2 \tilde{C} \pm \sqrt{6 \tilde{H}-2 \tilde{C}^{2}}\right)$. They correspond to the global extrema of $\operatorname{det} M(\phi)$ subject to equations (26).

We have two possibilities:

(1) the operator $M(\phi)$ has the same set of eigenvalues ( $\operatorname{say} \lambda_{1}^{+}, \lambda_{2}^{+}, \lambda_{2}^{+}$) for all critical angles $\phi$;

(2) there exist two critical angles, $\phi^{+}$and $\phi^{-}$such that the eigenvalues of $M\left(\phi^{\epsilon}\right)$ are $\lambda_{1}^{\epsilon}, \lambda_{2}^{\epsilon}, \lambda_{2}^{\epsilon}, \epsilon= \pm$, respectively.

Consider them separately.

(1) The only critical values of the function $\operatorname{det} M(\phi)$ are $\lambda_{1}^{+}\left(\lambda_{2}^{+}\right)^{2}$, the global maxima. Then $\operatorname{det} M(\phi)$ is constant and the eigenvalues of $M(\phi)$ are also constant: $\lambda_{1}^{+}, \lambda_{2}^{+}, \lambda_{2}^{+}$. It follows that the operator $\hat{M}(\phi): L \rightarrow L$ defined by $\hat{M}(\phi)=M(\phi)-$ $\lambda_{2}^{+} \mathrm{id}_{L}$ has eigenvalues $\lambda_{1}^{+}-\lambda_{2}^{+}, 0,0$ for all $\phi$, and in particular, $\operatorname{rk} \hat{M}(\phi)=1$. By (27), the matrix of $\hat{M}(\phi)$ in the basis $\left\{e_{i}\right\}$ has the form

$$
\begin{aligned}
\hat{M}(\phi)_{a b}= & \left(\lambda_{1}^{+}-\lambda_{2}^{+}\right) \delta_{a 3} \delta_{b 3} \cos ^{2} \phi \\
& +\left(R_{1 a 2 b}+R_{1 b 2 a}\right) \cos \phi \sin \phi+\left(R_{2 a 2 b}-\lambda_{2}^{+} \delta_{a b}\right) \sin ^{2} \phi,
\end{aligned}
$$

where $3 \leq a, b \leq 5$. Equating the coefficients of $\cos ^{3} \phi \sin \phi$ in $2 \times 2$-minors to zero, we find

$$
R_{1424}=R_{1425}+R_{1524}=R_{1525}=0 .
$$

It remains to show that $R_{1434}=R_{1535}=0$. The equations (28) are still true, if we replace the vectors $e_{4}, e_{5}$ by $e_{4}(\alpha)=\cos \alpha e_{4}+\sin \alpha e_{5}, e_{5}(\alpha)=-\sin \alpha e_{4}+\cos \alpha e_{5}$ 
lying in the $\lambda_{2}^{+}$-eigenspace of the operator $M\left(\phi_{0}\right)$. Then $R\left(e_{1}, e_{4}(\alpha), e_{3}, e_{4}(\alpha)\right)=$ $\cos 2 \alpha R_{1434}+\sin 2 \alpha\left(R_{1435}+R_{1534}\right) / 2$, and we can choose $\alpha$ in such a way that $R\left(e_{1}, e_{4}(\alpha), e_{3}, e_{4}(\alpha)\right)=0$. From the first equation of (24) it follows that $R\left(e_{1}, e_{5}(\alpha), e_{3}, e_{5}(\alpha)\right)=0$. Hence $e_{1}, e_{2}, e_{3}, e_{4}(\alpha), e_{5}(\alpha)$ is the sought basis.

(2) Since all the other possibilities are already considered, we can assume that for any initial choice of a critical two-plane $\sigma$, there exist two critical angles, $\phi^{+}$and $\phi^{-}$ such that the eigenvalues of the operator $M\left(\phi_{\epsilon}\right)$ are $\lambda_{1}^{\epsilon}, \lambda_{2}^{\epsilon}, \lambda_{2}^{\epsilon}, \epsilon= \pm$, respectively.

From the above, we know that if $e_{3}^{\epsilon}$ is a unit eigenvector of $M\left(\phi_{\epsilon}\right)$ corresponding to the eigenvalue $\lambda_{1}^{\epsilon}$, then both two-planes $\sigma^{\epsilon}=\operatorname{Span}\left(X\left(\phi^{\epsilon}\right), e_{3}^{\epsilon}\right), \epsilon= \pm$, are critical. Moreover, from (26) $\lambda_{1}^{\epsilon}+2 \lambda_{2}^{\epsilon}=\tilde{C},\left(\lambda_{1}^{\epsilon}\right)^{2}+2\left(\lambda_{2}^{\epsilon}\right)^{2}=\tilde{H}$ and so $\lambda_{1}^{+}+\lambda_{1}^{-}=\frac{2}{3} \tilde{C}=$ $\frac{2}{3}(C-\kappa(\sigma))$. Since $\lambda_{1}^{\epsilon}=\left\langle M\left(\phi^{\epsilon}\right) e_{3}^{\epsilon}, e_{3}^{\epsilon}\right\rangle=R\left(X\left(\phi^{\epsilon}\right), e_{3}^{\epsilon}, X\left(\phi^{\epsilon}\right), e_{3}^{\epsilon}\right)=\kappa\left(\sigma^{\epsilon}\right)$, we find that for every critical two-plane $\sigma$ there exist two critical two-planes, $\sigma^{+}, \sigma^{-}$ crossing $\sigma$ by a line and such that $\kappa\left(\sigma^{+}\right)+\kappa\left(\sigma^{-}\right)=\frac{2}{3}(C-\kappa(\sigma))$.

We say that two critical two-planes $\left(\sigma_{1}, \sigma_{2}\right)$, with sectional curvatures $\kappa\left(\sigma_{1}\right)=$ $x, \kappa\left(\sigma_{2}\right)=y$, form a critical pair, if they intersect by a line, and for a unit vector $X$ on that line, the eigenvalues of the operator $\left(R_{X}\right)_{\mid X^{\perp}}$ are $x, y, z, z$.

For any critical two-plane $\sigma$, the pairs $\left(\sigma, \sigma^{+}\right)$and $\left(\sigma, \sigma^{-}\right)$are critical with sectional curvatures $\left(\kappa(\sigma), \lambda_{1}^{+}\right)$and $\left(\kappa(\sigma), \lambda_{1}^{-}\right)$, respectively. Moreover, for any critical pair $\left(\sigma_{1}, \sigma_{2}\right)$ with sectional curvatures $(x, y)$, there exists another critical pair with sectional curvatures $(x, \tilde{y})$, where $\tilde{y}=\frac{2}{3}(C-x)-y$. Indeed, choose an orthonormal basis for $\mathbb{R}^{5}$ in such a way that $\sigma_{1}=\operatorname{Span}\left(e_{1}, e_{2}\right), \sigma_{2}=\operatorname{Span}\left(e_{1}, e_{3}\right)$. Denote $x=\kappa\left(\sigma_{1}\right), y=\kappa\left(\sigma_{2}\right), z, z$ the eigenvalues of the operator $\left(R_{e_{1}}\right)_{\mid e_{1}^{\perp}}$. For every $\phi$, the subspace $L=\sigma_{1}^{\perp}$ is an invariant subspace of the Jacobi operator $R_{X(\phi)}$, with $X(\phi)=\cos \phi e_{1}+\sin \phi e_{2}$. Define the operator $M(\phi): L \rightarrow L$ by $M(\phi)=\left(R_{X(\phi)}\right)_{\mid L}$. Since $\operatorname{Tr} M(\phi)$ and $\operatorname{Tr} M^{2}(\phi)$ are constant and the eigenvalues of $M(0)=\left(R_{e_{1}}\right)_{\mid L}$ are $y, z, z$, the function det $M(\phi)$ has a global extremum at $\phi=0$, and so the angle $\phi=0$ must be critical for det $M(\phi)$. Then there exists another critical angle $\tilde{\phi}$ such that the eigenvalues of $M(\tilde{\phi})$ are $\tilde{y}, \tilde{z}, \tilde{z}$, with $y+\tilde{y}=\frac{2}{3}(C-x)$. Moreover, for a unit eigenvector $\tilde{e}_{3}$ of $M(\tilde{\phi})$ corresponding to the eigenvalue $\tilde{y}$, the two-plane $\tilde{s}=\operatorname{Span}\left(X(\tilde{\phi}), \tilde{e}_{3}\right)$ is critical. This gives another critical pair $\left(\sigma_{1}, \tilde{\sigma}\right)$, with sectional curvatures $\left(x, \frac{2}{3}(C-x)-y\right)$.

If $\left(\sigma_{1}, \sigma_{2}\right)$ is a critical pair with sectional curvatures $(x, y)$, then the pair $\left(\sigma_{2}, \sigma_{1}\right)$ is also critical, with sectional curvatures $(y, x)$. It follows that starting with a critical pair with sectional curvatures $(x, y)$ we can successively construct a critical pair with sectional curvatures $(x, \tilde{y}), \tilde{y}=\frac{2}{3}(C-x)-y$, then a critical pair with sectional curvatures $(\tilde{y}, x)$, then a critical pair with sectional curvatures $(\tilde{y}, \tilde{x}), \tilde{x}=\frac{2}{3}(C-\tilde{y})-x$, and finally a critical pair with sectional curvatures $(\tilde{x}, \tilde{y})=\left(-\frac{5}{9} x+\frac{2}{3} y+\frac{2}{9} C, \frac{2}{3} C-\frac{2}{3} x-y\right)$.

If $\left(\sigma_{1}, \sigma_{2}\right)$ is a critical pair and $X$ is a unit vector in $\sigma_{1} \cap \sigma_{2}$, then the numbers $x$, $y, z, z$, the eigenvalues of $\left(R_{X}\right)_{\mid X^{\perp}}$, must satisfy the equations $x+y+2 z=C$, 
$x^{2}+y^{2}+2 z^{2}=H$ by (1). So the point $(x, y)$ lies on the ellipse

$$
3 x^{2}+3 y^{2}+2 x y-2 C(x+y)+\left(C^{2}-2 H\right)=0,
$$

in the $x y$-plane, and for some angle $\psi$ we have

$$
x=\frac{\sqrt{4 H-C^{2}}}{4}(\cos \psi+\sqrt{2} \sin \psi)+\frac{C}{4}, \quad y=\frac{\sqrt{4 H-C^{2}}}{4}(\cos \psi-\sqrt{2} \sin \psi)+\frac{C}{4} .
$$

The transformation $(x, y) \rightarrow(\tilde{x}, \tilde{y})=\left(-\frac{5}{9} x+\frac{2}{3} y+\frac{2}{9} C, \frac{2}{3} C-\frac{2}{3} x-y\right)$ corresponds to the shift $\psi \rightarrow \psi+\alpha$, with $e^{i \alpha}=-\frac{7}{9}+\frac{4 \sqrt{2}}{9} i$. This number is not a root of 1 . Indeed, for any $m \in \mathbb{N}, e^{m i \alpha}=9^{-m}\left(-a_{m}+\sqrt{2} b_{m} i\right)$, with $a_{m} \equiv b_{m} \equiv 1(\bmod 3)$, which can be easily proved by induction. So the set of pairs $(x, y)$, the sectional curvatures of critical pairs of two-planes, is dense on the ellipse (29). Then by compactness, for any two numbers $(x, y)$ satisfying (29) there exists a critical pair having sectional curvatures $(x, y)$. In particular, there exists a critical pair $\left(\sigma_{1}, \sigma_{2}\right)$ with $x=\kappa\left(\sigma_{1}\right)=\frac{1}{4} C \pm \frac{1}{2} \sqrt{12 H-3 C^{2}}$. Then, for a unit vector $X \in \sigma_{1} \cap \sigma_{2}$, the operator $\left(R_{X}\right)_{\mid \sigma_{1}^{\perp}}$ has an eigenvalue with multiplicity three, and we come to Case 1 with $\sigma=\sigma_{1}$.

From now on, we fix the basis $\left\{e_{i}\right\}$ constructed in Lemma 2. In this basis, the equations (22) hold, and we also have a symmetry with respect to permutations of $\left\{e_{2}, e_{3}, e_{4}, e_{5}\right\}$.

Introduce two $4 \times 4$-matrices, $T=\left(t_{b}^{a}\right)$ and $P=\left(P_{a b}\right)$ with entries

$$
t_{a}^{a}=0, t_{b}^{a}=R_{1 c b d}+R_{1 d b c}, \quad P_{a a}=0, P_{a b}=R_{a c a d},
$$

where $\{a, b, c, d\}=\{2,3,4,5\}$. We have, for any $a$,

$$
\sum_{f=2}^{5} t_{f}^{a}=0
$$

from the symmetries of the curvature tensor. Moreover, $P_{a b}+P_{b a}=\operatorname{Ric}_{c d}=0$ by (15), so $P$ is skew-symmetric.

Lemma 3. If $T=0$, then the sectional curvature is constant.

Proof. Let $T=0$. Then $R_{1 a b c}=0$ for all $a, b, c \geq 2$. Indeed, if $a=c$ this follows from (22). For $a, b, c$ pairwise nonequal, we have $R_{1 a b c}=\frac{1}{3}\left(t_{b}^{d}-t_{c}^{d}\right)$ by the first Bianchi identity.

From $R_{1 a b c}=0, a, b, c \geq 2$ and (22) we find that the equations (17) with $i=1$ and (19) with $i=1, j=a$ have the form

$$
\sum_{f=2}^{5} \kappa_{1 f}^{2}=H, \quad \sum_{f \neq a} \kappa_{1 f} \kappa_{a f}+\kappa_{1 a}^{2}=H,
$$

respectively. Summing up the second equation by $a$ from 2 to 5 and using the first equation and the fact that $\sum_{i=1}^{5} \kappa_{i j}=\operatorname{Ric}_{j j}=C$ we obtain $C^{2}=4 H$. 
It follows from (1) that for any $X \in \mathbb{R}^{5},\left(\operatorname{Tr} R_{X}\right)^{2}=4 \operatorname{Tr}\left(R_{X}^{2}\right)$. Hence $\left(R_{X}\right)_{\mid X^{\perp}}=$ $\frac{C}{4}\|X\|^{2} \operatorname{id}_{\mid X^{\perp}}$, that is, $R(X, U) X=\frac{C}{4}\|X\|^{2} U$ when $U \perp X$, and so the sectional curvature is constant.

Lemma 4. $R_{2345}=R_{2453}=R_{2534}=0$.

Proof. Using (22) we obtain from (18) with $j=1, i=a>1$ :

$$
\sum_{f=2}^{5} P_{a f} t_{a}^{f}=0 .
$$

The equation (20) with $j=1, i=a, k=b$ takes the form

$$
P_{a b} t_{b}^{a}-P_{b d} t_{a}^{d}-P_{b c} t_{a}^{c}+t_{a}^{b}\left(R_{a c b d}+R_{a d b c}\right)=0, \quad\{a, b, c, d\}=\{2,3,4,5\} .
$$

Introduce the numbers $\mu_{i}$ as follows:

$$
\mu_{0}=R_{2354}+R_{2453}, \quad \mu_{1}=R_{3254}+R_{3452}, \quad \mu_{2}=R_{4352}+R_{4253} .
$$

We have $R_{a c b d}+R_{a d b c}=\mu_{|a+b-7|}$ with $\{a, b, c, d\}=\{2,3,4,5\}$, and $\mu_{0}+\mu_{1}+$ $\mu_{2}=0$. Since $P_{b b}=t_{a}^{a}=0$, both (31) and (32) can be written in the form

$$
\sum_{f=2}^{5} P_{b f} t_{a}^{f}=P_{a b} t_{b}^{a}+\mu_{|a+b-7|} t_{a}^{b}, \quad a, b \geq 2 .
$$

Taking the sum by $a$ from 2 to 5 and applying (30) on the left hand side and (31) on the right hand side we come to $\sum_{a=2}^{5} \mu_{|a+b-7|} t_{a}^{b}=0$, for all $b \geq 2$. Using (30) and the fact that $t_{a}^{a}=0$, we solve for $t_{a}^{b}$ getting

$$
t_{a}^{b}=q^{b}\left(\mu_{|b+d-7|}-\mu_{|b+c-7|}\right)=3 q^{b} R_{b a c d}
$$

for some numbers $q^{2}, q^{3}, q^{4}, q^{5}$, where the permutation $(b, a, c, d) \rightarrow(2,3,4,5)$ is even (the last equation follows from $\mu_{|b+d-7|}=R_{b a d c}+R_{b c d a}$ and the first Bianchi identity).

Interchange $a$ and $b$ in (33) and subtract (31). Since $t_{b}^{b}=0$ and the matrix $P$ is skew-symmetric, we get $P_{a c}\left(t_{b}^{c}-t_{a}^{c}\right)+P_{a d}\left(t_{b}^{d}-t_{a}^{d}\right)=\mu_{|a+b-7|} t_{b}^{a}$, with the indices $c, d$ chosen in such a way that the permutation $(b, a, c, d) \rightarrow(2,3,4,5)$ is even. Substituting (34) we obtain $3 P_{a c} q^{c}\left(R_{c b a d}-R_{c a d b}\right)+3 P_{a d} q^{d}\left(R_{d b c a}-R_{d a b c}\right)=$ $\mu_{|a+b-7|} t_{b}^{a}$. But $R_{c b a d}-R_{c a d b}=-R_{b c a d}-R_{b d a c}=-\mu_{|a+b-7|}, R_{d b c a}-R_{d a b c}=$ $R_{a c b d}+R_{a d b c}=\mu_{|a+b-7|}$, and so

$$
\left(3 P_{a c} q^{c}-3 P_{a d} q^{d}+t_{b}^{a}\right) \mu_{|a+b-7|}=0, \quad(b, a, c, d) \text { an even permutation. }
$$

Now if $\mu_{|a+b-7|}\left(=\mu_{|c+d-7|}\right) \neq 0$, then

$$
\begin{aligned}
t_{b}^{a}=3 P_{a d} q^{d}-3 P_{a c} q^{c}, & t_{a}^{b}=3 P_{b c} q^{c}-3 P_{b d} q^{d}, \\
t_{d}^{c}=3 P_{c b} q^{b}-3 P_{c a} q^{a}, & t_{c}^{d}=3 P_{d a} q^{a}-3 P_{d b} q^{b},
\end{aligned}
$$


with the last three equations obtained by replacing the pair $(a, b)$ by $(b, a),(c, d)$, $(d, c)$, respectively, and taking into account the evenness of $(b, a, c, d)$. Multiplying these equations by $q^{a}, q^{b}, q^{c}, q^{d}$, respectively, and adding up, we get $q^{a} t_{b}^{a}+q^{b} t_{a}^{b}+$ $q^{c} t_{d}^{c}+q^{d} t_{c}^{d}=0$, since $P$ is skew-symmetric. By (34) this implies

$$
\left(\left(q^{a}\right)^{2}+\left(q^{b}\right)^{2}+\left(q^{c}\right)^{2}+\left(q^{d}\right)^{2}\right) R_{b a c d}=0 .
$$

If $R_{\text {bacd }} \neq 0$, then $T=0$ by (34) and the claim follows from Lemma 3. Assuming $T \neq 0$ we get that for any pair $a \neq b$ from $\{2,3,4,5\}$ either $R_{a c b d}+R_{a d b c}=$ $\mu_{|a+b-7|}=0$, or $R_{b a c d}=0$. From this and the first Bianchi identity we obtain $R_{2345}=R_{2453}=R_{2534}=0$.

Using the result of Lemma 4, the equations (22) and the definition of the $t_{a}^{b}$ 's and $P_{a b}$ 's we can simplify some of the equations (17)-(21): the equations (17) with $i=1$, (17) with $i=a$, (19) with $i=1, j=a$, (19) with $i=a, j=b$, (18) with $i=c$, $j=b$, and (20) with $i=1, j=a, k=b$ have the following form, respectively:

$$
\begin{gathered}
\sum_{p} \kappa_{1 p}^{2}=H, \\
\sum_{p} \kappa_{a p}^{2}+2 \sum_{f} P_{a f}^{2}=H, \\
\sum_{p} \kappa_{1 p} \kappa_{a p}+\kappa_{1 a}^{2}+\sum_{f}\left(t_{a}^{f}\right)^{2}=H, \\
\sum_{p} \kappa_{a p} \kappa_{b p}+\kappa_{a b}^{2}+\sum_{f<g} P_{f g}^{2}+3\left(P_{c d}^{2}-P_{a b}^{2}\right)+\left(t_{c}^{d}\right)^{2}+\left(t_{d}^{c}\right)^{2}=H, \\
P_{a d}\left(\kappa_{a c}-\kappa_{c d}\right)-P_{c d} P_{b d}-P_{c a} P_{b a}=0, \\
P_{c d}\left(\kappa_{1 c}-\kappa_{1 d}\right)+t_{b}^{a} t_{a}^{b}=0,
\end{gathered}
$$

where $\{a, b, c, d\}=\{2,3,4,5\}$.

Lemma 5. If $P=0$, then the sectional curvature is constant.

Proof. From (40) we get $t_{b}^{a} t_{a}^{b}=0$ for all $a, b \geq 2$. This, together with (30), implies that at least for one value of $a, t_{b}^{a}=0$ for all $b$. Without loss of generality, assume that $t_{3}^{2}=t_{4}^{2}=t_{5}^{2}=0$.

Summing up (38) by $b \neq a$ and adding (37) we obtain

$$
\sum_{i, p} \kappa_{a p} \kappa_{i p}+\sum_{f \neq a}\left(t_{a}^{f}\right)^{2}+\sum_{b \neq a}\left(\left(t_{d}^{c}\right)^{2}+\left(t_{c}^{d}\right)^{2}\right)=4 H,
$$

where $\{c, d\}=\{2,3,4,5\} \backslash\{a, b\}$. Now by (15) $\sum_{i} \kappa_{i j}=C$, so the first term on the left hand side equals $C^{2}$. The sum of the two remaining terms is $\sum_{g} \sum_{f \neq a}\left(t_{g}^{f}\right)^{2}=$ $\sum_{f, g}\left(t_{g}^{f}\right)^{2}-\sum_{g}\left(t_{g}^{a}\right)^{2}$. Therefore we obtain

$$
\sum_{g}\left(t_{g}^{a}\right)^{2}=\sum_{f, g}\left(t_{g}^{f}\right)^{2}+C^{2}-4 H .
$$

The right hand side does not depend on $a$. However, for $a=2$, the left hand side vanishes. Hence $T=0$ and it remains to apply Lemma 3 . 
From now on we assume that at least one of the $P_{a b}$ 's is nonzero.

Using (22) and Lemma 4 we simplify (18) with $i=b, j=1$ and (20) with $i=a$, $j=1, k=b$ to the form

$$
\begin{aligned}
& P_{b a} t_{b}^{a}+P_{b c} t_{b}^{c}+P_{b d} t_{b}^{d}=0, \\
& P_{a b} t_{b}^{a}-P_{b c} t_{a}^{c}-P_{b d} t_{a}^{d}=0,
\end{aligned}
$$

respectively, where $\{a, b, c, d\}=\{2,3,4,5\}$. Adding (41) and (42) we obtain

$$
P_{c b}\left(t_{a}^{c}-t_{b}^{c}\right)+P_{d b}\left(t_{a}^{d}-t_{b}^{d}\right)=0 .
$$

Interchanging $a \leftrightarrow b,(a, b) \leftrightarrow(c, d)$ and $(a, b) \leftrightarrow(d, c)$ we get, respectively:

$$
\begin{aligned}
& P_{c a}\left(t_{a}^{c}-t_{b}^{c}\right)+P_{d a}\left(t_{a}^{d}-t_{b}^{d}\right)=0, \\
& P_{a d}\left(t_{c}^{a}-t_{d}^{a}\right)+P_{b d}\left(t_{c}^{b}-t_{d}^{b}\right)=0, \\
& P_{a c}\left(t_{c}^{a}-t_{d}^{a}\right)+P_{b c}\left(t_{c}^{b}-t_{d}^{b}\right)=0 .
\end{aligned}
$$

So either $P_{c b} P_{d a}-P_{c a} P_{d b}=0$, or $t_{a}^{c}=t_{b}^{c}, t_{a}^{d}=t_{b}^{d}, t_{c}^{a}=t_{d}^{a}, t_{c}^{b}=t_{d}^{b}$.

Up to a sign, there are three minors of the form $P_{c b} P_{d a}-P_{c a} P_{d b}$ in the matrix $P$, depending on the choice of the pair $\{a, b\} \subset\{2,3,4,5\}$.

If at least two of them are nonzero, then $t_{a}^{c}=t_{b}^{c}=t_{d}^{c}$ for all $\{a, b, c, d\}=$ $\{2,3,4,5\}$ and so $T=0$ by (30). The proof is then completed with Lemma 3 .

Let precisely one of the three minors $P_{c b} P_{d a}-P_{c a} P_{d b}$ be nonzero, say $P_{23} P_{54}-P_{24} P_{53}=P_{23} P_{45}-P_{25} P_{43}=0$ and $P_{34} P_{25}-P_{24} P_{35} \neq 0$. Then $t_{4}^{2}=t_{5}^{2}, t_{5}^{3}=t_{4}^{3}, t_{3}^{4}=t_{2}^{4}, t_{3}^{5}=t_{2}^{5}$. Denoting $t^{2}=t_{4}^{2}, t^{3}=t_{5}^{3}, t^{4}=t_{3}^{4}, t^{5}=t_{3}^{5}$ we get $t_{3}^{2}=-2 t^{2}, t_{2}^{3}=-2 t^{3}, t_{5}^{4}=-2 t^{4}, t_{4}^{5}=-2 t^{5}$ from (30). From (43) with $a=4, b=3, c=1, d=2, P_{23} t^{2}=P_{53} t^{5}$. Then from (41) with $b=3$ we get $P_{32} t^{2}=P_{34} t^{4}$, and so $P_{23} t^{2}=P_{43} t^{4}=P_{53} t^{5}$. Similar arguments show that $P_{a b} t^{a}$ does not depend on $a \neq b$. In particular, $P_{53} t^{5}-P_{43} t^{4}=P_{52} t^{5}-P_{42} t^{4}=0$ and so $t^{4}=t^{5}=0$ since $P_{34} P_{25}-P_{24} P_{35} \neq 0$. Similarly, $t^{2}=t^{3}=0$, that is, $T=0$, and it remains to apply Lemma 3.

Finally, assume that all the minors $P_{c b} P_{d a}-P_{c a} P_{d b}$ vanish. It is easy to see that $P_{c b} P_{d a}=0$ for all $\{a, b, c, d\}=\{2,3,4,5\}$, and so the matrix $P_{a b}$ is of one of the following forms, up to relabelling the subscripts:

$$
\left(\begin{array}{cccc}
0 & P_{23} & P_{24} & P_{25} \\
-P_{23} & 0 & 0 & 0 \\
-P_{24} & 0 & 0 & 0 \\
-P_{25} & 0 & 0 & 0
\end{array}\right), \quad\left(\begin{array}{cccc}
0 & P_{23} & P_{24} & 0 \\
-P_{23} & 0 & P_{34} & 0 \\
-P_{24} & -P_{34} & 0 & 0 \\
0 & 0 & 0 & 0
\end{array}\right)
$$

and at least one of the $P_{a b}$ 's is nonzero. 
Lemma 6. If the matrix $P$ has the form (44, $)$, then at most one of the $P_{2 a}, a>2$ can be nonzero.

Proof. Taking $a=2$ in (39) we obtain

$$
P_{2 d}\left(\kappa_{2 c}-\kappa_{d c}\right)=P_{2 c} P_{2 b}, \quad\{b, c, d\}=\{3,4,5\} .
$$

First assume none of $P_{23}, P_{24}, P_{25}$ vanishes.

Then we get $\kappa_{2 c}-\kappa_{d c}=\kappa_{2 b}-\kappa_{d b}$ for any triple $\{b, c, d\}=\{3,4,5\}$. Introduce the numbers $v_{3}, v_{4}, v_{5}$ by $v_{d}=\kappa_{2 c}-\kappa_{d c}, c \neq 2, d$. We have $\kappa_{d c}=\kappa_{2 c}-v_{d}=\kappa_{2 d}-v_{c}$ and so $\kappa_{2 c}+v_{c}, c \neq 2$ does not depend on $c$. Denote $\xi=\kappa_{2 c}+v_{c}$. Then

$$
\kappa_{2 c}=\xi-v_{c}, \quad \kappa_{d c}=\xi-v_{c}-v_{d}, \quad c, d \neq 2, c \neq d .
$$

Then by (15), $C=\sum_{i} k_{2 i}=\kappa_{12}+3 \xi-\sum_{f} v_{f}$ and for $a \neq 2, C=\sum_{i} k_{a i}=$ $\kappa_{1 a}+3 \xi-2 v_{a}-\sum_{f} v_{f}$. It follows that $k_{1 a}=k_{12}+2 v_{a}$, and so by (15), $C=$ $\sum_{i} k_{1 i}=4 \kappa_{12}+3 \sum_{f} v_{f}$. Then

$$
\kappa_{12}=\xi-\sum_{f} v_{f}, \quad \kappa_{1 a}=\xi-\sum_{f} v_{f}+2 v_{a}, \quad a \neq 2 .
$$

Substituting this and $\left(46,44_{1}\right)$ to $(35,36)$ we obtain $2 P_{2 a}^{2}+4 \xi^{2}-4 \xi \sum_{f} v_{f}+$ $2 \sum_{f} v_{f}^{2}+2 v_{a}^{2}=4 \xi^{2}-4 \xi \sum_{f} v_{f}+4 \sum_{f} v_{f}^{2}=H$, and so

$$
P_{2 a}^{2}=v_{b}^{2}+v_{c}^{2}, \quad\{a, b, c\}=\{3,4,5\} .
$$

On the other hand, $(45,46)$ imply

$$
P_{2 d} v_{d}=P_{2 c} P_{2 b}, \quad\{d, b, c\}=\{3,4,5\} .
$$

It follows that $P_{2 a}^{2}=\left(\frac{P_{2 a} P_{2 c}}{P_{2 b}}\right)^{2}+\left(\frac{P_{2 a} P_{2 b}}{P_{2 c}}\right)^{2}$ and so $\left(\frac{P_{2 c}}{P_{2 b}}\right)^{2}+\left(\frac{P_{2 b}}{P_{2 c}}\right)^{2}=1$. Since the left hand side must be greater than or equal to 2 , we come to the contradiction.

So at least one of $P_{23}, P_{24}, P_{25}$ vanishes, and the claim follows from (45).

Lemma 7. If the matrix $P$ has the form $\left(44_{2}\right)$, then either the sectional curvature is constant, or at most one of the $P_{2 a}, a>2$ is nonzero.

Proof. Assume that all three numbers $P_{23}, P_{24}, P_{34}$ are nonzero (otherwise, relabelling the subscripts we come to a subcase of $\left.\left(44_{1}\right)\right)$.

The equation (43) with $d=5$ gives $P_{c b}\left(t_{a}^{c}-t_{b}^{c}\right)=0,\{a, b, c\}=\{2,3,4\}$, and so $t_{a}^{c}=t_{b}^{c}$. Denote $t^{c}=t_{a}^{c}=t_{b}^{c},\{a, b, c\}=\{2,3,4\}$

Taking $d=5$ in (40) we find $t_{a}^{b} t_{b}^{a}=0$. Therefore $t^{b} t^{a}=0$ for all $a, b \neq 5$, and so at least two of the three numbers $t^{2}, t^{3}, t^{4}$ vanish. Let say $t^{3}=t^{4}=0$. Then also $t^{2}=0$ by (41) with $a=2, b=3, c=4, d=5$. Hence $t_{a}^{2}=t_{a}^{3}=t_{a}^{4}=0$ for all $a$. 
The equation (40) with $c, d \neq 5$ gives $\kappa_{12}=\kappa_{13}=\kappa_{14}$. Denote their common value by $\kappa$. From (35) and (36) with $a=5$ we get $3 \kappa^{2}=\kappa_{25}^{2}+\kappa_{35}^{2}+\kappa_{45}^{2}$. But from (15) $C=\sum_{i} \kappa_{1 i}=\sum_{i} \kappa_{5 i}$, hence $3 \kappa=\kappa_{25}+\kappa_{35}+\kappa_{45}$. It follows that $\kappa_{25}=\kappa_{35}=\kappa_{45}=\kappa$. Applying (15) again we find that $\kappa_{23}=\kappa_{24}=\kappa_{34}$. Denote their common value by $\hat{\kappa}$.

The equation (37) with $a \neq 5$ now yields $2 \kappa \hat{\kappa}+\kappa^{2}+\kappa \kappa_{15}+\left(t_{a}^{5}\right)^{2}=H$. It follows that $\pm t_{2}^{5}= \pm t_{3}^{5}= \pm t_{4}^{5}$ and so $t_{2}^{5}=t_{3}^{5}=t_{4}^{5}=0$ by (30). Thus $T=0$ and the claim follows from Lemma 3.

As Lemma 6 and Lemma 7 show, it remains to consider the case when only one of the $P_{a b}$ 's is nonzero. After relabelling we can assume that $P_{23} \neq 0$, and all the other $P_{a b}$ 's vanish.

The equation (39) with $(b, c)=(4,5),(5,4)$ yields

$$
\kappa_{34}=\kappa_{24}, \quad \kappa_{35}=\kappa_{25}, \quad \kappa_{12}=\kappa_{13},
$$

the latter equation follows from (15): $C=\sum_{i} \kappa_{2 i}=\sum_{i} \kappa_{3 i}$.

From (42) we get $t_{a}^{2}=t_{a}^{3}=0$ for all $a$, and from (40) with $a=4, b=5$ and (47) $t_{5}^{4} t_{4}^{5}=0$. Without loss of generality assume $t_{4}^{5}=0$. Then (21) with $i=1, j=2$, $k=3, l=4$ takes the form

$$
P_{23}\left(t_{3}^{4}-t_{2}^{4}\right)+t_{2}^{5}\left(\kappa_{12}+\kappa_{34}\right)+t_{3}^{5}\left(\kappa_{13}+\kappa_{24}\right)=0 .
$$

But $t_{2}^{5}=-t_{3}^{5}$ by (30), so (47) implies $t_{3}^{4}=t_{2}^{4}$.

From the equations (36) with $a=2$ and (38) with $a=2, b=3$ we find

$$
\kappa_{12}^{2}+\kappa_{23}^{2}+\kappa_{24}^{2}+\kappa_{25}^{2}+2 P_{23}^{2}=\sum_{i} \kappa_{2 i} \kappa_{3 i}+\kappa_{23}^{2}-2 P_{23}^{2}+\left(t_{5}^{4}\right)^{2}=H,
$$

and so $4 P_{23}^{2}=\left(t_{5}^{4}\right)^{2}$ by (47). Then $2 P_{23}^{2}= \pm t_{5}^{4}$ and we can take the minus sign on the right hand side replacing the vector $e_{1}$ by $-e_{1}$, if necessary. It now follows from (30) that the only nonzero entries of the matrices $P$ and $T$ can be

$$
t_{3}^{4}=t_{2}^{4}=\mu, \quad t_{5}^{4}=-2 \mu, \quad t_{3}^{5}=v, \quad t_{2}^{5}=-v, \quad P_{23}=\mu \neq 0 .
$$

Then from (35), (36) with $a=4$, and (37) with $a=4$ we get

$\kappa_{12}^{2}+\kappa_{13}^{2}+\kappa_{14}^{2}+\kappa_{15}^{2}=\kappa_{14}^{2}+\kappa_{24}^{2}+\kappa_{34}^{2}+\kappa_{45}^{2}=\kappa_{12} \kappa_{24}+\kappa_{13} \kappa_{34}+\kappa_{14}^{2}+\kappa_{15} \kappa_{45}=H$,

so the vectors $\left(\kappa_{12}, \kappa_{13}, \kappa_{14}, \kappa_{15}\right)$ and $\left(\kappa_{24}, \kappa_{34}, \kappa_{14}, \kappa_{45}\right)$ are equal. Combining this with (47) we find:

$$
\kappa_{12}=\kappa_{13}=\kappa_{24}=\kappa_{34}, \quad \kappa_{35}=\kappa_{25}, \quad \kappa_{15}=\kappa_{45} .
$$

Now from (15), $C=\sum_{i} \kappa_{4 i}=\sum_{i} \kappa_{5 i}$, which gives $\kappa_{14}+2 \kappa_{24}=\kappa_{15}+2 \kappa_{25}$, and from (36) with $a=4, a=5, H=\sum_{i} \kappa_{4 i}^{2}=\sum_{i} \kappa_{5 i}^{2}$ and so $\kappa_{14}^{2}+2 \kappa_{24}^{2}=\kappa_{15}^{2}+2 \kappa_{25}^{2}$. 
Hence either $\kappa_{14}=\kappa_{15}, \kappa_{24}=\kappa_{25}$ or $\kappa_{24}=\frac{1}{4} \kappa_{14}+\frac{3}{4} \kappa_{15}, \kappa_{25}=\frac{3}{4} \kappa_{14}+\frac{1}{4} \kappa_{15}$. But the first case is not possible, since otherwise (37) with $a=5$ and (49) imply $\kappa_{12}^{2}+\kappa_{13}^{2}+\kappa_{14}^{2}+\kappa_{15}^{2}+4\left(t_{5}^{4}\right)^{2}=H$, and so $t_{5}^{4}=0$ by (35). This contradicts to $\mu \neq 0$ from (48). In the second case, we solve (15) getting

$$
\begin{gathered}
\kappa_{12}=\kappa_{13}=\kappa_{23}=\kappa_{24}=\kappa_{34}=\alpha-\gamma, \\
\kappa_{25}=\kappa_{35}=\alpha-3 \gamma, \quad \kappa_{15}=\kappa_{45}=\alpha, \quad \kappa_{14}=\alpha-4 \gamma,
\end{gathered}
$$

where $C=4 \alpha-6 \gamma$.

Substituting (50) and (48) to (21) with $i=1, j=2, k=3, l=5$ we find $v=3 \gamma$. From $(50,35,36)$ we get $4 \alpha^{2}-12 \alpha \gamma+18 \gamma^{2}=4 \alpha^{2}-12 \alpha \gamma+12 \gamma^{2}+2 \mu^{2}=H$, and so $\mu= \pm \sqrt{3} \gamma$. Replacing the vector $e_{5}$ by $-e_{5}$, if necessary, we can take $\mu=\sqrt{3} \gamma$.

Using (48) and the fact that $R_{1 a b c}=\frac{1}{3}\left(t_{b}^{d}-t_{c}^{d}\right)$, which follows from the definition of the $t_{b}^{a}$ 's we find that the nonzero components of the algebraic curvature tensor $R$ are those listed in (50) and

$$
\begin{gathered}
R_{1234}=\gamma, \quad R_{1235}=\sqrt{3} \gamma, \quad R_{1324}=-\gamma, \quad R_{1325}=\sqrt{3} \gamma, \\
R_{1423}=-2 \gamma, \quad R_{2425}=\sqrt{3} \gamma, \quad R_{3435}=-\sqrt{3} \gamma .
\end{gathered}
$$

It can be checked directly that the algebraic curvature tensor with components given by $(50,51)$ satisfies the first two Ledger formulae (1).

\section{References}

[1] J. Berndt, F. Tricerri, L. Vanhecke, Generalized Heisenberg groups and Damek-Ricci harmonic spaces. Lecture Notes in Math. 1598, Springer-Verlag, Berlin 1995. Zbl 0818.53067 MR 1340192

[2] A. Besse, Manifolds all of whose geodesics are closed. Ergeb. Math. Grenzgeb. 93, Springer-Verlag, Berlin, Heidelberg, New York 1978. Zbl 0387.53010 MR 0496885

[3] G. Besson, G. Courtois, S. Gallot, Volumes, entropies et rigidités des espaces localement symétriques de courbure strictement négative. C. R. Acad. Sci. Paris Sér. I Math. 319 (1994), 81-84. Zbl 0812.53041 MR 1285903

[4] E. Damek, F. Ricci, A class of nonsymmetric harmonic Riemannian spaces. Bull. Amer. Math. Soc. 27 (1992), 139-142. Zbl 0755.53032 MR 1142682

[5] J.-H. Eschenburg, A note on symmetric and harmonic spaces. J. London Math. Soc. (2) 21 (1980), 541-543. Zbl 0441.53042 MR 0577728

[6] P. Gilkey, A. Swann, L. Vanhecke, Isoparametric geodesic spheres and a conjecture of Osserman concerning the Jacobi operator. Quart. J. Math. Oxford (2) 46 (1995), 299-320. Zbl 0848.53023 MR 1348819

[7] A. Gray, L. Vanhecke, Riemannian geometry as determined by the volumes of small geodesic balls. Acta Mathematica 142 (1979), 157-198. Zbl 0428.53017 MR 0521460 
[8] S. Helgason, Differential geometry, Lie groups, and symmetric spaces. Pure Appl. Math. 80, Academic Press, New York, San Francisco, London 1978. Zbl 0451.53038 MR 0514561

[9] A. J. Ledger, Symmetric harmonic spaces. J. London Math. Soc. 32 (1957), 53-56. Zbl 0084.37406 MR 0083796

[10] K. Ramachandran, A. Ranjan, Harmonic manifolds with some specific volume densities. Proc. Indian Acad. Sci. Math. Sci. 107 (1997), 251-261. Zbl 0903.53030 MR 1467430

[11] A. Ranjan, An intrinsic approach to Lichnerowicz conjecture. Proc. Indian Acad. Sci. Math. Sci. 110 (2000), 27-34. Zbl 0952.53022 MR 1746369

[12] A. Ranjan, H. Shah, Harmonic manifolds with minimal horospheres. J. Geom. Anal. 12 (2002), 683-694. Zbl 01983425 MR 1916864

[13] Z. I. Szabo, The Lichnerowicz Conjecture on harmonic manifolds. J. Differential Geom. 31 (1990), 1-28. Zbl 0686.53042 MR 1030663

[14] Z. I. Szabo, Spectral theory for operator families. In Differential geometry. Part 3: Riemannian geometry, Proc. Symp. Pure Math. 54 (1993) (part 3), 615-665. Zbl 0791.58100 MR 1216651

[15] A. G. Walker, On Lichnerowicz's conjecture for harmonic 4-spaces. J. London Math. Soc. 24 (1949), 21-28. Zbl 32.18801 MR 0030280

[16] Y. Watanabe, The sectional curvature of a 5-dimensional harmonic Riemannian manifold. Kodai Math. J. 6 (1983), 100-109. Zbl 0519.53013 MR 0698331

[17] T. J. Willmore, Riemannian geometry. Oxford Science Publications, The Clarendon Press, Oxford University Press, New York 1993. Zbl 0797.53002 MR 1261641

Received September 14, 2000; revised January 14, 2003

Y. Nikolayevsky, Department of Mathematics, La Trobe University, Bundoora, Victoria 3083, Australia

E-mail: Y.Nikolayevsky@latrobe.edu.au 